\title{
Correlation of histological findings from a large ciliochoroidal melanoma with CT perfusion and 3T MRI dynamic enhancement studies
}

\author{
Jose S Pulido' \\ Norbert G Campeau ${ }^{2}$ \\ Ernst Klotz ${ }^{3}$ \\ Andrew N Primak ${ }^{2}$ \\ Osama Saba ${ }^{3}$ \\ Kaan Gunduz' \\ Herbert Cantrill ${ }^{5}$ \\ Diva Salomão ${ }^{1,4}$ \\ Cynthia $\mathrm{H}$ McCollough ${ }^{2}$ \\ 'Department of Ophthalmology; \\ ${ }^{2}$ Department of Radiology; ${ }^{3}$ Siemens \\ Medical Solutions, Malvern, PA, USA; \\ ${ }^{4}$ Department of Laboratory Medicine \\ and Pathology, Mayo Clinic College \\ of Medicine, Rochester, MN, USA; \\ ${ }^{5}$ VitreoRetinal Surgery, Minneapolis, \\ MN, USA
}

\begin{abstract}
Background: The initial use of a 64-slice computed tomography (CT) scanner for obtaining quantitative perfusion data from a large ciliochoroidal melanoma, and correlation with 3T magnetic resonance imaging (MRI) dynamic enhancement and tumor histology.

Methods: The CT perfusion scan was performed using $80 \mathrm{kVp}, 250 \mathrm{~mA}$ and 1-sec rotation time for $40 \mathrm{sec}$. The analysis was performed using commercial perfusion analysis software with a prototype 3-dimensional motion correction tool. Dynamic contrast-enhanced 3-Tesla MRI measured the kinetics of enhancement to estimate the vascular permeability. The timedependent enhancement patterns were obtained using the average signal intensity using Functool analysis software. The involved globe was enucleated and microscopic evaluation of the tumor was performed.

Results: The perfusion parameters blood flow, blood volume and permeability surface area product in the affected eye determined by CT perfusion analysis were $118 \mathrm{ml} / 100 \mathrm{ml} / \mathrm{min}$, $11.3 \mathrm{ml} / 100 \mathrm{ml}$ and $48 \mathrm{ml} / 100 \mathrm{ml} / \mathrm{min}$. Dynamic MRI enhancement showed maximal intensity increase of $111 \%$. The neoplasm was a ciliochoroidal spindle cell melanoma which was mitotically active (13 mitoses/40 hpf). Vascular loops and arcades were present throughout the tumor. The patient developed metastases within 9 months of presentation.

Conclusion: Quantitative CT perfusion analysis of ocular tumors is feasible with motion correction software.

Keywords: ciliochoroidal melanoma, CT perfusion imaging, MR enhancement imaging, tumor blood volume, tumor blood flow, tumor permeability
\end{abstract}

\section{Introduction}

Choroidal melanomas have an incidence of 5-7 new cases per one million persons per year (Shields et al 1992; Singh et al 2003). They occur much more commonly in Caucasians than in African Americans (Egan et al 1988). There are no known risk factors other than a family history or ocular melanocytosis (Singh et al 1998).

In the eye, choroidal melanomas cause visual loss. Typical sites of metastases are the liver, the lung and rarely other organs (COMS Report No. 15 2001).

The risk factors for metastasis have traditionally been related to the size of the tumor and the cell type (McLean et al 1983; COMS Report No. 18 2001). Another and very important risk factor for metastasis is monosomy 3 , although the mechanism of how this is related to increased metastases is still not known (Prescher et al 1996).

In addition, PAS positive loops are an independent risk factor for the development of metastasis (Rummelt et al 1995). These loops have been shown to be vascular loops made of a combination of endothelial cells and tumor cells. An increased number of vascular loops correlate with a worse prognosis.
Correspondence: Cynthia H McCollough Dept. of Raiology, Mayo Clinic College of Medicine, 200 First Street SW, Rochester, MN 55904, USA

Tel + I 507266063 I

Fax +I 5072842405

Email mccollough.cynthia@mayo.edu 
Aside from the size and location, or cytogenic abnormality (monosomy 3 status) and gene expression profiling information derived from fine need aspiration, all of the other risk factors are usually known only at the time of the enucleation of the eye following examination of the specimen (Gunduz et al 1999; COMS Report No. 18 2001).

Since vascularity may be an important and independent indicator for prognosis, methods to determine the amount of vascularity short of removal of the eye are needed. Mueller and associates (2002) have attempted to look at the vascularity by using indocyanine green (ICG). Buerk and colleagues (2004) have attempted to evaluate intratumoral vascularity with magnetic resonance imaging (MRI) by determining the relative enhancement over time. The problem with this technique is that it is only a relative technique and does not allow absolute quantitation. Additionally, the temporal sampling of the enhancing signal was only approximately 40 seconds using the MRI dynamic enhancement protocol. We elected this approach to match the technique previously used by Buerk and colleagues (2004) to allow comparison of the computed tomography (CT) perfusion results with previously studied methods. It is important to note, however, MRI perfusion techniques can sample at 1-1.5 images per second, similar to CT.

Once metastasis occurs, the death rate is $80 \%$ at one year and $92 \%$ at two years (Bedikian et al 1995). Since the uveal melanomas express vascular endothelial growth factor (VEGF), which helps the tumor attract vessels to help nourish the tumor, attempts at the use of VEGF inhibitors are being considered as well (Lee et al 2006).

The use of CT to quantify vascular perfusion within tumors and organs has only recently been developed (Dugdale et al 1999; Seppenwoolde et al 2000; Fournier et al 2004; Kiessling et al 2004; Kan and Kobayashi et al 2005; Kan and Phongkitkarun et al 2005; Gandhi et al 2006; Laghi 2006). The benefits of CT perfusion include the ability to measure absolute perfusion values and high temporal resolution (measurements can be acquired every $1 \mathrm{sec}$, or less). Dependent on the acquisition protocol and analysis software various quantitative perfusion indices may be calculated. The goal of this study was to develop an in vivo measure of vascularity for patients presenting with choroidal melanomas, with the ultimate hope being that this will provide improved prognostic information without removal of the affected eye. We report for the first time, in a patient with a large choroidal melanoma, the results of a 64-slice CT perfusion study and compare it to the relative enhancement from MRI and histopathologic findings.

\section{Materials and methods}

\section{Patient selection}

A 50-year old patient presented with decreased vision in the right eye. Past medical and ocular history was unremarkable. The visual acuity was $20 / 50$ and $20 / 20$ in the right and left eye respectively. The patient was noted to have a large pigmented ciliochoroidal melanoma with a base of $19 \times 16 \mathrm{~mm}$. Standardized echography showed the presence of a low reflective lesion with a thickness of $10.7 \mathrm{~mm}$. Systemic evaluation including liver function tests, PET/CT scanning showed no evidence of detectable metastases. A diagnosis of large primary ciliochoroidal melanoma was made. Therapeutic alternatives were discussed and an enucleation was chosen. The patient agreed to participate in a study protocol designed to evaluate the correlation of $\mathrm{CT}$ perfusion and MR enhancement indicators of tumor vascularity with histopathological findings. Imaging was performed prior to surgical enucleation of the involved globe. This HIPPA compliant study was approved by our Institutional Review Board. At 9 months following initial presentation, the patient developed diffuse metastatic disease.

\section{CT scan protocol}

CT imaging was performed on a 64-slice CT scanner (Sensation 64, Siemens Medical Solutions, Forchheim, Germany). The patient was placed in the scanner bore in a head-first position, lying on his side, with his head tilted comfortably toward the affected side to approximately $15^{\circ}$. This position was used in order to keep the unaffected eye outside of the prescribed scan volume for the perfusion acquisition. Cushions and straps were placed to assist the patient in maintaining this position comfortably. To accurately verify patient radiation exposure, a thermoluminescent dosimeter (TLD) was placed on the surface of the closed eyelid of the unaffected eye and another placed adjacent to affected eye. A tissue-equivalent gel pad (SuperFlab, MedTec, Orange City, IA) was placed over the unaffected eye to help reduce the radiation to the lens of the unaffected eye.

A CT radiograph was acquired and used to center the scan volume over the affected eye while maximizing the distance to the unaffected eye. The superior ophthalmic artery and cavernous portion of the internal carotid artery ipsilateral to the affected eye were included in the scan field. These vessels were used to measure the arterial input functions used for the perfusion calculations. The patient was coached to keep the affected (open) eye fixed on one location, marked on the gantry in front of the patient's head. This helped to minimize motion of the eyeball during the 40 -second duration of the 
perfusion scan, which could cause motion misregistration errors in the data analysis. Parameters for the perfusion scan included: scan field-of-view $(\mathrm{SFOV})=50 \mathrm{~cm}$, perfusion scan mode (ie, no table increment between each scan), detector collimation $=24 \times 1.2 \mathrm{~mm}$, tube potential $=80$ $\mathrm{kVp}$, tube-current time product $=250 \mathrm{mAs}$, reconstruction FOV $150 \mathrm{~mm}, 1$ second tube rotation time for 40 seconds. The perfusion scan was initiated $3 \mathrm{sec}$ after the start of the injection of $40 \mathrm{cc}$ of iodinated contrast material (Omnipaque 350, Amersham, Cork, Ireland), administered intravenously at $5 \mathrm{cc} / \mathrm{sec}$, followed by an injection of an additional $30 \mathrm{cc}$ of iodinated contrast material at $1 \mathrm{cc} / \mathrm{sec}$ and a $30 \mathrm{cc}$ of saline injected at $1 \mathrm{cc} / \mathrm{sec}$. Data was reconstructed using a $2.4 \mathrm{~mm}$ image widths for perfusion analysis. All raw data were saved for post-processing and perfusion analysis.

\section{MRI dynamic enhancement technique}

The MRI enhancement study was done using a similar technique to that described previously (Mueller et al 2002). MRI with intravenous contrast enhancement was performed on a 3.0 Tesla whole-body scanner (Signa VHi 3T; General Electric Medical Systems, Milwaukee, WI, USA). Sagittal T1-weighted FLAIR (fluid attenuated inversion recovery) images $\left(\mathrm{TR}=2,613 \mathrm{~ms}, \mathrm{TE}=16 \mathrm{~ms}, \mathrm{FOV}=24 \mathrm{~cm}^{2}\right.$, acquisition matrix $=512 \times 192$, image thickness $=5 \mathrm{~mm}$ ) through the head were used for graphic prescription of axial T2weighted fast-spin echo images $(\mathrm{TR}=5,000 \mathrm{~ms}, \mathrm{TE}=102 \mathrm{~ms}$, $\mathrm{FOV}=15 \mathrm{~cm}^{2}$, acquisition matrix $=416 \times 416$, image thickness $=1.5 \mathrm{~mm}$, gap $=0.5 \mathrm{~mm}$ ) through the orbits. Precontrast axial T1-weighted spin echo images (TR $=400 \mathrm{~ms}$, $\mathrm{TE}=19 \mathrm{~ms}, \mathrm{FOV}=14 \mathrm{~cm}^{2}$, acquisition matrix $=416 \times 160$, image thickness $=3 \mathrm{~mm}$, gap $=0.5 \mathrm{~mm}$ ) were then obtained through the ocular lesion. For lesions placed in the superior or inferior regions of the globe, images were obtained in the coronal plane for improved visualization. Following intravenous administration of gadolinium contrast (Omniscan, $0.1 \mathrm{mmol} / \mathrm{kg}$ ) via an antecubital vein, five axial T1-weighted spin echo sections were acquired through the globes at 1minute intervals for 7 minutes.

\section{Histopathology}

The patient's eye was surgically enucleated. The specimen was fixed for 48 hours in 10\% buffered formalin and then gross examination was performed. The specimen was embedded in paraffin and sectioned at $4 \mu$. The sections were stained with hematoxylin-eosin and periodic acid/Schiff. The eye was examined by light microscopy for tumor location, cell type, mitotic index, presence of vascular loops and arcades and presence of extraocular extension.

\section{Data analysis}

A commercially available, FDA-approved CT perfusion software package (Neuro PCT, Siemens Medical Solutions, Forchheim, Germany) was used for analysis of the CT perfusion data. In order to correct for some motion of the orbit during the scan acquisition, a prototype $3 \mathrm{D}$ registration tool was used to re-register the orbit to the original coordinates.

Perfusion images of the motion-corrected central $2.4 \mathrm{~mm}$ section were used to obtain quantitative measures of tumor blood flow, tumor blood volume and vascular permeability. The arterial input function was measured from an artery within the scan field. Blood flow (F) (ml/100 ml tissue/min) was calculated using the maximum slope model (Miles et al 1991; Klotz et al 1999), and blood volume (ml/100 ml tissue) (BV) and volume transfer coefficient (Ktrans) $(\mathrm{ml} / 100 \mathrm{ml}$ tissue/min) using a modified Patlak algorithm (Patlak et al 1983; Dugdale et al 1999; Ng et al 2006). The volume transfer coefficient Ktrans from the slope of the Patlak fit describes the portion of whole blood flow that is extracted into extravascular space: Ktrans $=\mathrm{E}^{*} \mathrm{~F}$, with $\mathrm{E}$ the extraction fraction. This differs from Ktrans as it is now used in MRI, which is normalized to plasma flow by a factor of (1-Hct), with Hct being the hematocrit value. Permeability surface area product (PS) was calculated using the Renkin-Crone equation of capillary transport which provides a relation between E, F and PS: E = (1 - exp(-PS/F(1-Hct)) $)($ Renkin 1959; Crone 1963).

The MRI data was analyzed using the method previously described in detail (Mueller et al 2002). The time-dependent enhancement patterns were obtained using the average signal intensity from five separate regions of interest placed over each of three locations: the ocular lesion, the brain gray matter, and the extraocular muscles, as a function of time (Functools; General Electric Medical Systems, Milwaukee, WI, USA). The average and standard deviation of the maximum enhancement for each region were calculated as the percentage difference between the pre-enhancement and maximum post-enhancement signal intensities divided by the pre-enhancement signal intensity for each tissue on the T1-weighted images (Microsoft Excel; Microsoft Corporation, Redmond, WA, USA). The gray matter of the brain served as a measure of signal intensity stability over time, because no enhancement should occur away from blood vessels when the blood-brain barrier is intact. The muscle 
served as a measure of enhancement expected for normally perfused tissue.

\section{Results}

The results of the CT perfusion study were based on tumor voxel time-attenuation curves (TAC) such as the one shown in the lower half of Figure 1. TAC curves are readily measured with a CT scanner and reflect the change in X-ray attenuation (CT number, HU) as the injected iodinated contrast material flows through the tumor. The corresponding Patlak analysis is shown in the upper half of Figure 1. The average perfusion parameters within the choroidal melanoma were determined by region of interest analysis (Figure 2). Blood flow was 118 $\mathrm{ml} / 100 \mathrm{ml} / \mathrm{min}$, blood volume $11.3 \mathrm{ml} / 100 \mathrm{ml}$ and Ktrans $62 \mathrm{ml} / 100 \mathrm{ml} / \mathrm{min}$. The resulting permeability surface area product was $48 \mathrm{ml} / 100 \mathrm{ml} / \mathrm{min}$.

The MRI enhancement showed a maximum enhancement of $111 \%$ over baseline levels at $150 \mathrm{sec}$. The slope was 2.37 MR enhancement units/sec (Figure 3a). There was a slow wash-out of 0.66 units/sec. There appeared to be a correlation between the areas of increased perfusion by $\mathrm{CT}$ and increased enhancement by MR (Figures 2, 3b).

Histology showed the presence of a large spindle cell melanoma with no sign of extraocular extension (Figures $4 \mathrm{a}, 4 \mathrm{~b}$ ). The neoplasm was mitotically active (13 units/40 hpf). Vascular loops and arches were present throughout the tumor. There was no tumor necrosis or tumor infiltration of lymphocytes. The overlying retina was atrophic, and there was a localized exudative detachment.

\section{Discussion}

Present techniques for the diagnosis of choroidal melanoma do not allow determination of vascular flow. Considering that both brachytherapy and possibly anti-angiogenic therapy affect the vascularity of the tumor, it is important to develop methods that can determine the perfusion characteristics of choroidal melanomas.

Relative MRI enhancement and absolute CT perfusion imaging studies may allow the noninvasive evaluation of the vascularity of tumors. These techniques have been used to look at tumors in other organs (Seppenwoolde et al 2000; Fournier et al 2004; Laghi 2006). This is the first study of CT perfusion studies in ocular tumors. One advantage of using CT compared to MRI is better spatial resolution $\left(0.29 \times 0.29 \times 2.4 \mathrm{~mm}^{3}\right.$ voxel size for CT and $0.63 \times 0.83 \times 3.0 \mathrm{~mm}^{3}$ voxel size for MR). It would be very challenging from a technical standpoint to perform MRI enhancement studies of the orbit with such high spatial resolution. Furthermore, the temporal resolution of CT (we used 1 second intervals between measurements although this could be as short as 0.33 seconds) is superior to that of MRI perfusion techniques which has best temporal resolution of approximately 1 second. The potential drawback of using $\mathrm{CT}$ is that the patient is exposed to ionizing radiation, which has been shown to induce lens opacities at doses above 500 mGy and cataracts at doses above 5000 mGy (International Commission on Radiological Protection 1991). For this study, we sought to minimize dose to the unaffected eye by tilting the head and using a superficial absorber. With this technique, the dose to the surface of the unaffected eye was $120 \mathrm{mGy}$, which is well below the thresholds for induction of lens opacities or cataracts. Thus, CT offers advantages over MRI in spatial and temporal resolution with negligible risk of radiation injury to the unaffected eye. Furthermore, the CT technique can provide absolute quantitative measurements of perfusion, whereas the MRI technique provides relative values that are not able to be compared between patients. This lack of an absolute measure limits the correlation of histology findings to tumor perfusion for prognostic purposes.

It is important to note, however, that the dynamic MR enhancement technique used in this study gives different and complementary information about the tumor compared with the CT perfusion technique. The CT perfusion technique used in this study relies on intravascular indicator dilution analysis and two-compartment modeling, which may be used to characterize blood flow, blood volume and vascular permeability of the tumor. The MRI dynamic enhancement technique provides an estimate of the degree of endovascular permeability of the intrinsic tumor vascularity (Mueller et al 2002). The clinical significance of these measures of tumor vascularity remains to be further elucidated though interestingly, in this patient, there was fast development of metastatic disease. One hypothesis would be that more rapidly growing tumors have higher vascular permeability than that of slower growing. Interestingly, in this case, there was close correlation in the location and degree of increased perfusion in the tumor with the area of increased enhancement (permeability) (Figures 2, 3b).

This particular tumor had a marked number of small complex vessels forming loops and arcs by histological evaluation. CT perfusion studies have already been used as a predictive determinant of whether a lesion in the lung or liver is cancerous (Seppenwoolde et al 2000; Fournier et al 2004; Laghi 2006). It also has been used in patients before and after treatment to determine if there was a response to therapy. It also has been used to determine the effect of antiangiogenic 


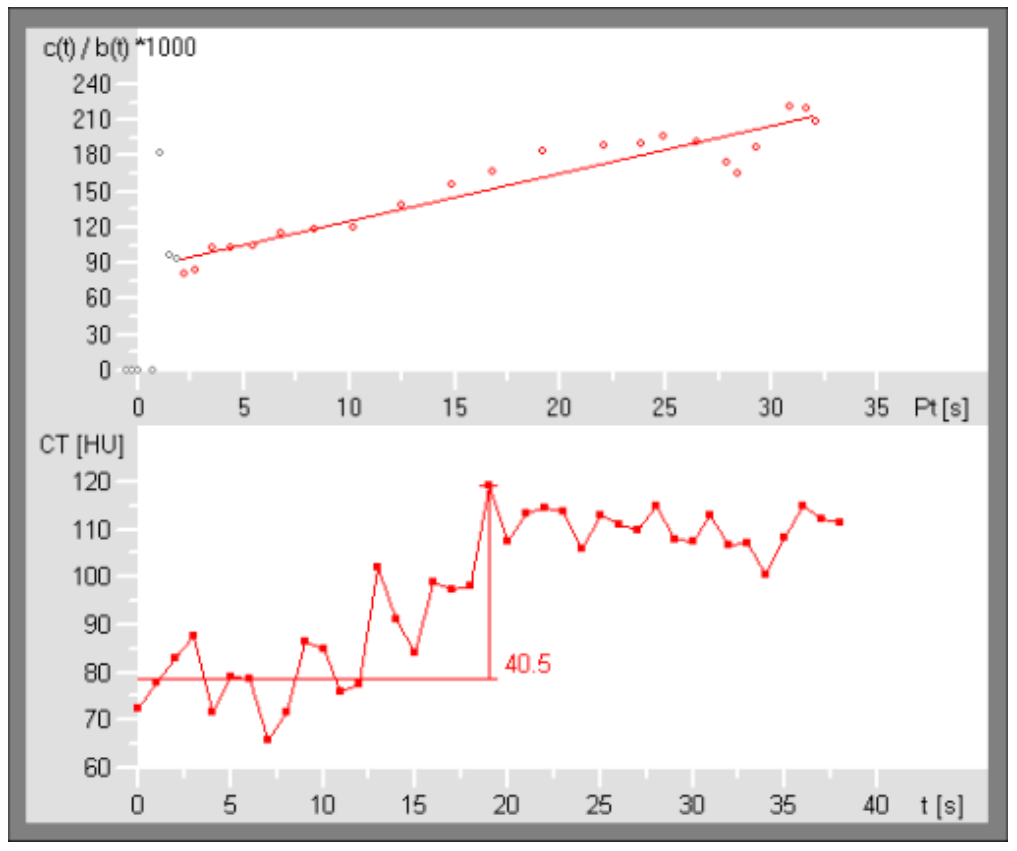

Figure I CT perfusion analysis is based on tumor voxel time-attenuation curves (TAC) such as the one shown in the lower half. Plotted is the CT-number (HU) as a function of time in seconds. For the voxel selected, the TAC starts at about $80 \mathrm{HU}$ and increases by about $40 \mathrm{HU}$. The corresponding Patlak analysis is shown in the upper half. Plotted is the enhancement in the tumor voxel relative to the enhancement in the reference artery $(c(t)) /(b(t))$ as a function of the so called Patlak time $(\mathrm{Pt})$.

agents on tumor blood flow parameters. Considering that VEGF receptors have been noted on iris and choroidal melanomas, future studies to determine if chemotherapeutic agents including antiangiogenic therapies may have an effect on the tumors will require the use of diagnostic methods that help to determine the blood flow parameters to the lesion (Lee et al 2006). In addition, it may be an earlier determinant of metastases since CT perfusion has been reported to have a high sensitivity in predicting malignancy of solitary pulmonary nodules and liver lesions (Kiessling et al 2004; Kan et al 2005). Furthermore, this technique has been shown to have a potential in assessing the grade, activity and treatment response of lymphoma (Dugdale et al 1999). Perfusion values of liver metastases as measured with CT perfusion have been described to correlate with patient survival (Kan et al 2005), whereas in head-and-neck cancer, CT perfusion appeared to be an independent predictor of local outcome after irradiation (Gandhi et al 2006). Functional CT perfusion

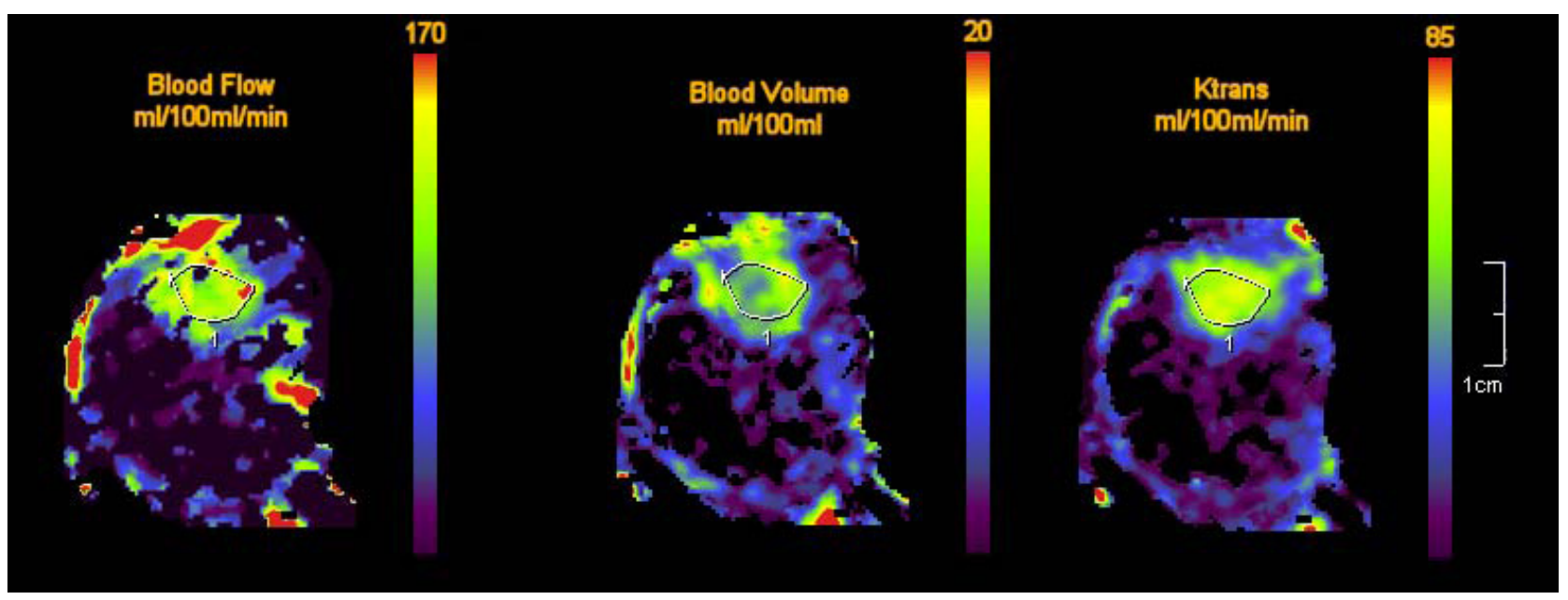

Figure $2 \mathrm{CT}$ perfusion parameter images. Shown from left to right: Blood flow (I I8 ml//00 ml/min), blood volume (II.3 ml/I00 ml) and Ktrans (62 ml/I00 ml/min). Numbers are averaged over the displayed ROI. The color scale maps numbers from 0 to the number displayed above the scale. 


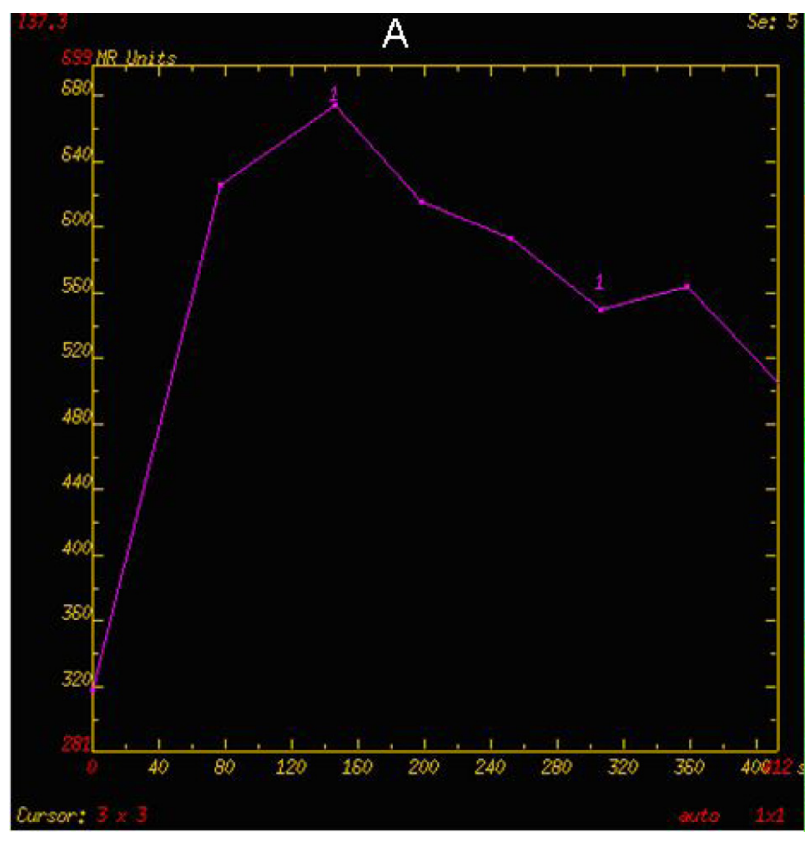

Figure 3a Dynamic MRI enhancement. Relative MRI signal enhancement obtained from a region of interest placed within the choroidal melanoma (see ROI on image below) as a function of time (shown on $\mathrm{x}$-axis in seconds). Following administration of gadolinium contrast via an antecubital vein, 7 volumes of same $\mathrm{TI}$-weighted spin echo images were acquired through the globes at I-minute intervals for 7 minutes. Data was analyzed on an independent computer workstation using Functool software.

techniques have previously been successful in demonstrating decreasing effects of different angiogenesis inhibitors on tumor blood flow parameters (Kan and Kobayashi et al 2005; Kan and Phongkitkarun et al 2005; Wong et al 2006). The use of this technique before and after successful radiotherapy may add further data as to the validity of the thought that brachytherapy affects the tumor by ablating its vascularity (Kaiserman et al 2004). Future studies should be performed to

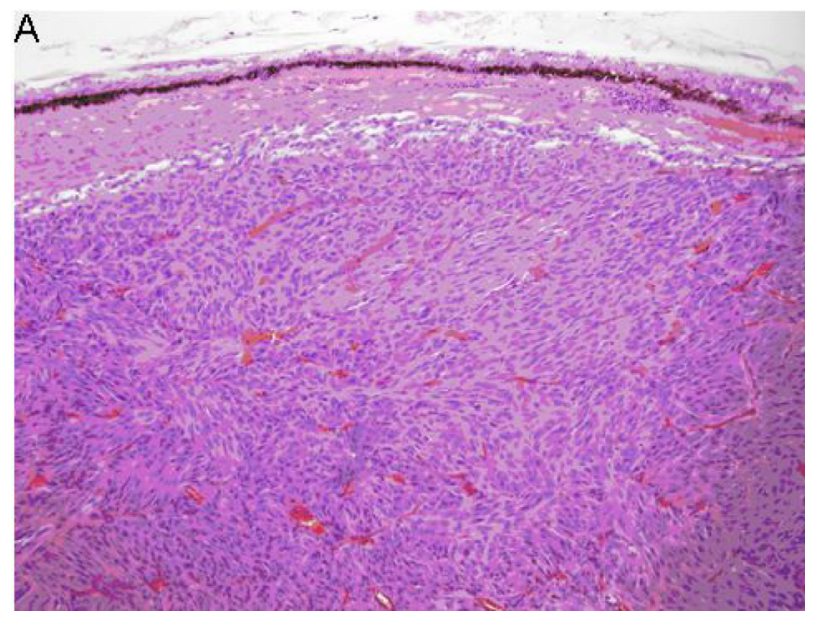

Figure 4a Low power photomicrograph of the tumor showing a predominance of cells forming fascicles and marked vascular channels. (Hematoxylin and eosin. X200).

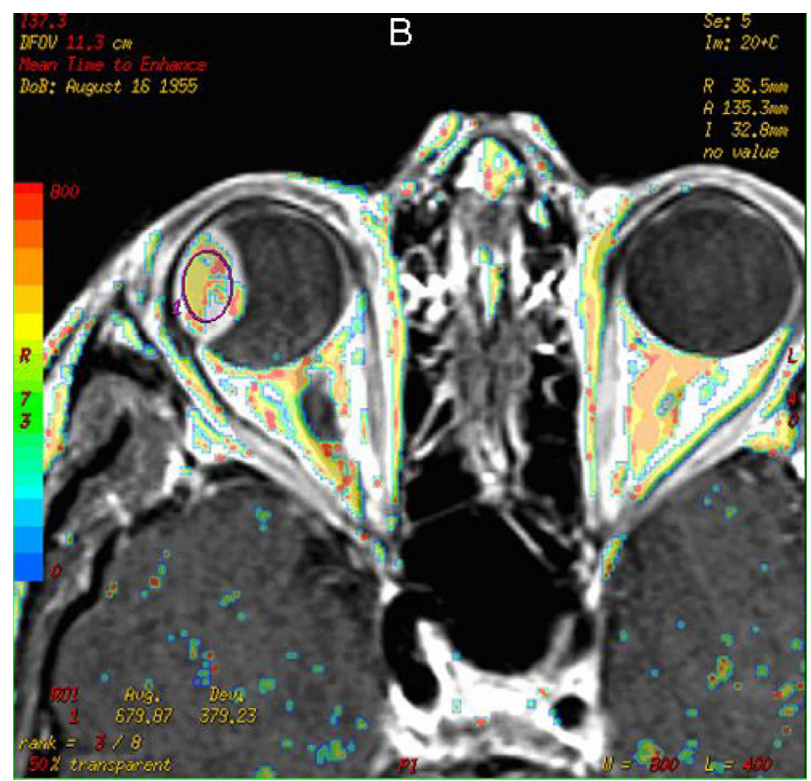

Figure 3b MRI dynamic enhancement color overlay. The area under the dynamic enhancement curves was integrated on a pixel-by-pixel basis and overlayed onto its corresponding gadolinium enhanced axialTI-weighted image. Regions of the choroidal tumor which showed more enhancement over time are represented by the hotter colors. This dynamic enhancement is related to tumor permeability, and there is good correlation with CT perfusion results (see Figure 2).

compare perfusion with other available techniques, including monosomy 3 testing and ICG studies, as well as to determine the clinical value and cost-effectiveness of each technique.

In summary, we present the first attempt at determining the perfusion characteristics of a large choroidal melanoma using state of the art 64-slice CT perfusion. We estimated tumor permeability using dynamic enhancement MRI. In addition, we show the relationship to histology of the tumor.

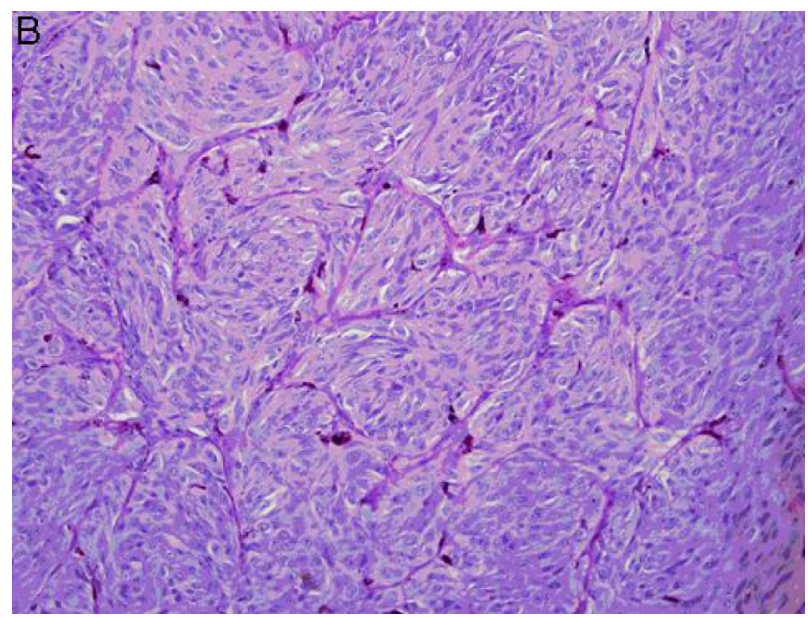

Figure 4b High power photomicrograph showing several mitotic figures and the PAS positive vascular arcs and loops (Periodic acid Schiff X400). 


\section{Acknowledgments}

The authors would like to thank the following colleagues for their assistance and support: Edward P Lindell, MD, Robert J Witte, MD, John I Lane, MD, J Douglas Cameron, MD, John Woog, MD, Glen Sturchio, MS, Chris Tipka, RT, Theresa A Nielsen, and Kristina M Nunez.

\section{References}

Bedikian AY, Legha SS, Mavligit G, et al. 1995. Treatment of uveal melanoma metastatic to the liver: a review of the M. D. Anderson Cancer Center experience and prognostic factors. Cancer, 76:1665-70.

Buerk BM, Pulido JS, Chiong I, et al. 2004. Vascular perfusion of choroidal melanoma by 3.0 tesla magnetic resonance imaging. Trans Am Ophthalmol Soc, 102:209-15; discussion 215-17.

COMS Report No. 15. 2001. Assessment of metastatic disease status at death in 435 patients with large choroidal melanoma in the Collaborative Ocular Melanoma Study (COMS). Arch Ophthalmol, 119:670-6.

Coms Report No. 18. 2001. The COMS randomized trial of iodine 125 bracytherapy for choroidal melanoma, III. Initial mortality findings. Arch Ophthalmol, 119:969-82.

Crone C. 1963. The permeability of capillaries in various organs as determined by use of the 'Indicator Diffusion' Method. Acta Physiol Scand, 58:292-305.

Dugdale PE, Miles KA, Bunce I, et al. 1999. CT measurement of perfusion and permeability within lymphoma masses and its ability to assess grade, activity, and chemotherapeutic response. J Comput Assist Tomogr, 23:540-7.

Egan KM, Seddon JM, Glynn RJ, et al. 1988. Epidemiologic aspects of uveal melanoma. Surv Ophthalmol, 32:239-51.

Fournier LS, Cuenod CA, de Bazelaire C, et al. 2004. Early modifications of hepatic perfusion measured by functional CT in a rat model of hepatocellular carcinoma using a blood pool contrast agent. Eur Radiol, $14: 2125-33$.

Gandhi D, Chepeha DB, Miller T, et al. 2006. Correlation between initial and early follow-up CT perfusion parameters with endoscopic tumor response in patients with advanced squamous cell carcinomas of the oropharynx treated with organ-preservation therapy. AJNR Am J Neuroradiol, 27:101-6.

Gunduz K, Shields CL, Shields JA, et al. 1999. Radiation retinopathy following plaque radiotherapy for posterior uveal melanoma. Arch Ophthalmol, 117:609-14.

International Commission on Radiological Protection. 1991. 1990 Recommendations of the International Commission on Radiological Protection. New York, NY.

Kaiserman I, Anteby I, Chowers I, et al. 2004. Post-brachytherapy initial tumour regression rate correlates with metastatic spread in posterior uveal melanoma. Br J Ophthalmol, 88:892-5.
Kan Z, Kobayashi S, Phongkitkarun S, et al. 2005a. Functional CT quantification of tumor perfusion after transhepatic arterial embolization in a rat model. Radiology, 237:144-50.

Kan Z, Phongkitkarun S, Kobayashi S, et al. 2005b. Functional CT for quantifying tumor perfusion in antiangiogenic therapy in a rat model. Radiology, 237:151-8.

Kiessling F, Boese J, Corvinus C, et al. 2004. Perfusion CT in patients with advanced bronchial carcinomas: a novel chance for characterization and treatment monitoring? Eur Radiol, 14:1226-33.

Klotz E, Konig M. 1999. Perfusion measurements of the brain: using dynamic CT for the quantitative assessment of cerebral ischemia in acute stroke. Eur J Radiol, 30:170-84.

Laghi A. 2006. Multidetector CT (64 Slices) of the liver: examination techniques. Eur Radiol, 17:675-83.

Lee ES, Baratz KH, Pulido JS, et al. 2006. Expression of vascular endothelial growth factor in iris melanoma. Arch Ophthalmol, 124:1349-50.

McLean IW, Foster WD, Zimmerman LE, et al. 1983. Modifications of Callender's classification of uveal melanoma at the Armed Forces Institute of Pathology. Am J Ophthalmol, 96:502-9.

Miles KA, Hayball M, Dixon AK. 1991. Colour perfusion imaging: a new application of computed tomography. Lancet, 337:643-5.

Mueller AJ, Freeman WR, Schaller UC, et al. 2002. Complex microcirculation patterns detected by confocal indocyanine green angiography predict time to growth of small choroidal melanocytic tumors: MuSIC Report II. Ophthalmology, 109:2207-14.

Ng QS, Goh V, Fichte H, et al. 2006. Lung cancer perfusion at multi-detector row CT: reproducibility of whole tumor quantitative measurements. Radiology, 239:547-53.

Patlak CS, Blasberg RG, Fenstermacher JD. 1983. Graphical evaluation of blood-to-brain transfer constants from multiple-time uptake data. J Cereb Blood Flow Metab, 3:1-7.

Prescher G, Bornfeld N, Hirche H, et al. 1996. Prognostic implications of monosomy 3 in uveal melanoma. Lancet, 347:1222-5.

Renkin EM. 1959. Transport of potassium-42 from blood to tissue in isolated mammalian skeletal muscles. Am J Physiol, 197:1205-10.

Rummelt V, Folberg R, Woolson RF, et al. 1995. Relation between the microcirculation architecture and the aggressive behavior of ciliary body melanomas. Ophthalmology, 102:844-51.

Seppenwoolde Y, Muller SH, Theuws JC, et al. 2000. Radiation dose-effect relations and local recovery in perfusion for patients with non-small-cell lung cancer. Int J Radiat Oncol Biol Phys, 47:681-90.

Shields JA, Shields CL. 1992. Intraocular tumors: A text and atlas. Philadelphia: Saunders, pp. 119-205.

Singh A, Topham A. 2003. Incidence of uveal melanoma in the United States: 1973-1997. Ophthalmology, 110:956-61.

Singh AD, De Potter P, Fijal BA, et al. 1998. Lifetime prevalence of uveal melanoma in white patients with oculo(dermal) melanocytosis. Ophthalmology, 105:195-8.

Wong CY, Savin M, Sherpa KM, et al. 2006. Regional yttrium-90 microsphere treatment of surgically unresectable and chemotherapy-refractory metastatic liver carcinoma. Cancer Biother Radiopharm, 21:305-13. 
